\title{
Novel mutations in SLC3OA2 involved in the pathogenesis of transient neonatal zinc deficiency
}

\author{
Naoya Itsumura', Yoshie Kibihara ${ }^{2}$, Kazuhisa Fukue ${ }^{1}$, Akiko Miyata ${ }^{3}$, Kenji Fukushima ${ }^{4,8}$, Risa Tamagawa-Mineoka ${ }^{5}$, \\ Norito Katoh ${ }^{5}$, Yukina Nishito' ${ }^{1}$, Riko Ishida ${ }^{1}$, Hiroshi Narita' ${ }^{2}$, Hiroko Kodama ${ }^{6,7}$ and Taiho Kambe ${ }^{1}$
}

BACKGROUND: Infants are vulnerable to zinc deficiency. Thus, abnormally low breast milk zinc levels cause transient neonatal zinc deficiency (TNZD) in breast-fed infants. TNZD has been considered to be rare because of a paucity of citations in the published literature. However, recent studies of affected mothers identified four missense mutations in the solute carrier family 30 member 2 gene (SLC3OA2), which encodes the zinc transporter, ZnT2.

METHODS: Genetic analyses of SLC3OA2/ZnT2 in three Japanese mothers secreting low-zinc milk (whose infants developed TNZD) were performed. The effects of identified mutations were examined in a cell-based assay. Furthermore, 31 single-nucleotide polymorphisms (SNPS) in SLC3OA2/ZnT2 were evaluated for their potential involvement in low-zinc levels in milk.

RESULTS: Each mother had a different novel heterozygous mutation in SLC30A2/ZnT2. One mutation reduced splicing efficiency of the SLC3OA2/ZnT2 transcript, and all ZnT2 mutants were defective in zinc transport and were unstable in cells. Moreover, four SNPs caused a significant loss of zinc-transport activity, similar to that in disease-causing ZnT2 mutants.

CONCLUSION: Our results indicate that many SLC30A2/ZnT2 mutations cause or potentially cause TNZD. Genetic information concerning TNZD pathogenesis is limited, and our results suggest that the TNZD frequency may be higher than previously thought.

A pproximately $10 \%$ of all human proteins are thought to be zinc-binding, and zinc plays a pivotal role as a structural, catalytic, and signaling component of proteins in numerous physiological processes $(1,2)$. Thus, zinc in breast milk is essential for the normal growth and development of infants $(3,4)$. To meet infants' requirements, large amounts of zinc $(1-3 \mathrm{mg} / \mathrm{d})$ are secreted into breast milk during lactation, particularly in the first 3 mo after birth (5-7). This is considerably higher than levels in the maternal serum (8). A reduction in the level of breast milk zinc causes breast-fed infants to develop zinc deficiency, which presents with a broad range of defects, such as erythematous and erosive dermatitis, persistent diarrhea, hair loss, immune system dysfunction, and retardation in physical development $(1,8-17)$. It is well known that the demand for zinc increases rapidly in thriving preterm infants $(18,19)$, and thus a risk of zinc deficiency increases in preterm infants $(20,21)$. However, symptomatic zinc deficiency has been found even in full-term infants, mostly because of inherited disorders.

Inherited zinc deficiency disorders in breast-fed full-term infants are classified into two types: acrodermatitis enteropathica (AE) (Online Mendelian Inheritance in Man (OMIM) 201100) and transient neonatal zinc deficiency (TNZD) (OMIM 608118) $(1,16,17,19,22,23)$. AE is caused by the intestinal malabsorption of zinc, and as a result, infants still suffer from zinc deficiency after weaning. In contrast, TNZD is caused by low zinc concentrations in breast milk; thus, infants develop the symptoms of zinc deficiency only during breastfeeding. TNZD, as its name suggests, does not reoccur after weaning, and is therefore definitively different from $\mathrm{AE}$. Mutations in zinc transporter genes have been identified to be responsible for each of these disorders $(1,16,17)$; $\mathrm{AE}$ is caused by mutations in the solute carrier family 39 member 4 gene (SLC39A4) (also known as the Zrt- and Irt-like protein 4 gene (ZIP4)), while TNZD is caused by mutations in SLC30A2 (also known as the $\mathrm{Zn}$ transporter 2 gene $(Z n T 2))$. ZIP4 is a zinc importer on the apical membrane of enterocytes and is essential for zinc absorption. Thus, disease-causing ZIP4 mutants lead to severe zinc deficiency. Conversely, ZnT2 mobilizes zinc into the secretory vesicles in mammary epithelial cells, which is thought to be released into the alveolar lumen, and then secreted into breast milk during lactation $(5,6)$. Hence, disease-causing SLC30A2/ZnT2 mutations cause mothers to produce zinc-deficient breast milk, and infants fed with this milk acquire severe zinc deficiency.

Over 30 mutations in SLC39A4/ZIP4 have been found to cause AE; however, there is a paucity of citations related to

\footnotetext{
'Division of Integrated Life Science, Graduate School of Biostudies, Kyoto University, Kyoto, Japan; ${ }^{2}$ Department of Food Science, Kyoto Women's University, Kyoto, Japan; ${ }^{3}$ Saiwai Pediatric Clinic, Tokyo, Japan; ${ }^{4}$ Department of Pediatrics, San-in Rosai Hospital, Yonago, Japan; ${ }^{5}$ Department of Dermatology, Graduate School of Medical Science, Kyoto Prefectural University of Medicine, Kyoto, Japan; ${ }^{6}$ Department of Pediatrics, School of Medicine, Teikyo University, Tokyo, Japan; ${ }^{7}$ Department of Health and Dietetics, Faculty of Health and Medical Sciences, Teikyo Heisei University, Tokyo, Japan; ${ }^{8}$ Current affiliation: Department of Pediatrics, Tottori University Hospital, Yonago, Japan. Correspondence:Taiho Kambe (kambe1@kais.kyoto-u.ac.jp)

Received 15 March 2016; accepted 22 March 2016; advance online publication 15 June 2016. doi:10.1038/pr.2016.108
} 
TNZD in the literature, leading to the assumption that TNZD is extremely rare. Indeed, it has not been possible to define the frequency of this disorder (24). However, in the past $8 \mathrm{y}$, at least 20 different Japanese case reports and abstracts have presented zinc deficiency in full-term infants caused by zinc-deficient breast milk (see Supplementary Table S1 online). Since the first finding of a missense mutation in SLC30A2/ZnT2 as a genetic cause of TNZD by Kelleher's group (10), one nonsense and four missense mutations have been identified in mothers secreting zinc-deficient breast milk, whose infants developed TNZD (12-15). In this article, we analyzed genomic DNA from three of the mothers included in the 20 Japanese reports and identified three novel loss-of-function SLC30A2/ZnT2 mutations. Moreover, we report here that four single-nucleotide polymorphisms (SNPs) in SLC30A2/ZnT2, which result in amino acid substitutions, cause ZnT2 to lose its zinc-transport activity. Our results provide crucial genetic information concerning TNZD pathogenesis in breast-fed infants.

\section{RESULTS}

\section{Three Novel, Missense, Loss-of-Function, Heterozygous SLC30A2/ZnT2 Mutations}

For this study, we recruited three Japanese mothers who produced zinc-deficient breast milk and whose breast-fed infants suffered from severe zinc deficiency (see Supplementary Table S2 online). We analyzed all SLC30A2/ZnT2 exons and their flanking regions, including splice sites, in each mother and found three novel heterozygous missense mutations: c. $838 \mathrm{G}>\mathrm{A}$ at the end of exon 6 (with reference to the adenine of the start methionine as +1 ), c.935C $>\mathrm{T}$ (p.T312M) in exon 7, and c.1063G $>$ C (p.E355Q) in exon 8 (see Figure 1a,b and Supplementary Table S2 online). The c.838G >A mutation (a $\mathrm{G}$ to A substitution) conserved the consensus sequence of the splice site (human U2-type intron) (25), but our reverse transcriptase-PCR analysis using ZnT2 cDNA constructs containing intron 6 (Figure 2a) indicated that it reduced the splicing efficiency of the $Z n T 2$ transcript to almost one-fourth that of the wild-type (WT) sequence (Figure 2b). Thus, the c.838G $>$ A mutation results in reduced splicing efficiency of intron 6 and causes substitution p.G280R in the ZnT2 protein when intron 6 is spliced out.

\section{Evaluation of Zinc-Transport Activity of the Three Novel ZnT2 Mutants Found in Japanese Mothers}

We previously evaluated the defects of four TNZD missense ZnT2 mutations (two of which we identified (W152R and S296L) (13) and two of which were found by others (H54R and G87R) $(10,12)$ ), using genetically engineered mutant $\left(\mathrm{ZnT1}^{---} \mathrm{MT}^{-/-} \mathrm{ZnT4^{-/- }}\right)$ cells (13). These cells show a zinc-sensitive phenotype, which is reversed by the stable expression of zinc-transport competent ZnT2. Similar to the well-established mutant BHK cell line (26), our cell system is simple but useful for the examination of ZnT2 zinc-transport activity.

In the present study, the stable expression of G280R, T312M, and E355Q ZnT2 mutants failed to reverse the zinc-sensitive phenotype of $\mathrm{ZnT1}^{-/-} \mathrm{MT}^{-/-} \mathrm{ZnT4} 4^{-/-}$cells, suggesting that these mutants have impaired zinc-transport activity (Figure 3a). Moreover, lack of zinc-transport activity in each mutant was confirmed using a fluorescent zinc-selective probe, Zinpyr-1 (27). Zinpyr-1 can detect vesicular/compartmentalized zinc; therefore, in this study, its fluorescence reflects zinc transported by ZnT2 from the cytosol into the vesicles. Zinc mobilized into the secretory vesicles by ZnT2 is thought to be released into the alveolar lumen in lactating mammary glands $(5,6)$. The cells used above were cultured in the presence of 30 $\mu \mathrm{mol} / \mathrm{Z} \mathrm{ZnSO}_{4}$ for $48 \mathrm{~h}$, loaded with Zinpyr-1, and the fluorescence intensity was analyzed by flow cytometry. WT ZnT2 expression increased the fluorescence intensity of Zinpyr-1 in $\mathrm{ZnT1}^{-/-} \mathrm{MT}^{-1-} \mathrm{ZnT4^{-/ }}$ cells cultured in the presence of 30 $\mu \mathrm{mol} / 1 \mathrm{ZnSO}_{4}$ (Figure 3c). However, the expression of G280R, $\mathrm{T} 312 \mathrm{M}$, or E355Q ZnT2 mutants produced no increase in fluorescence intensity (Figure 3c). Taken together, these findings indicate that all three mutations result in a lack of zinc-transport activity.

As detected in other previously reported TNZD-causing mutants (13), the protein stability of these three ZnT2 mutants was significantly reduced (Figure 4a). Moreover, immunoprecipitation analysis indicated that all three ZnT2 mutants can form dimers with WT ZnT2 (Figure 4b), suggesting that they may impair the zinc-transport functions of WT ZnT2 in a dominant negative manner. These results indicate that SLC30A2/ZnT2 c.838G>A (p.G280R), c.935C >T (p.T312M), and c.1063G $>C$ (p.E355Q) mutations cause loss-of-function and that haploinsufficiency or possible dominant negative effects result in low levels of zinc in the breast milk of affected mothers. Reduced splicing efficiencies may also contribute to pathogenesis in the $c .838 \mathrm{G}>\mathrm{A}$ mutation case.

\section{Evaluation of SNPs on ZnT2 Zinc-Transport Function}

We next evaluated the effects of SLC30A2/ZnT2 SNPs archived in the NCBI public database (http://www.ncbi.nlm.nih.gov/ snp) on ZnT2 zinc-transport activity to determine their potential involvement in the pathogenesis of TNZD. For a first evaluation, we randomly selected 31 SLC30A2/ZnT2 SNPs (all of which are shown to be minor alleles with frequencies up to 0.0036) (see Table 1 and Supplementary Table $\mathbf{3} 3$ online) that cause amino acid substitutions, and investigated their effects in viability assays using $Z n T 1^{-1-} M T^{--} Z n T 4^{-1-}$ cells. We found that four SNPs: rs148861822, c.542C >T (p.T181M); rs200520278, c.567C >A (p.N189K); rs201084300, c.698G>A (p.G233D); and rs377192955, c.1063G>A (p.E355K), failed to reverse the zinc-sensitive phenotype of $Z n T 1^{-/} M T^{--} Z n T 4^{-l-}$ cells (Table 1 and Figure 3b). Flow cytometry analysis using Zinpyr-1 confirmed that these SNPs caused ZnT2 to lose zinc-transport activity (Figure 3d), as in the case of TNZD-causing mutations (see Figure 3c). These SNP mutants were all destabilized but could form dimers with WT ZnT2, similar to the TNZDcausing mutants described above (Figure 5a,b). These results suggest that the four SNPs cause mothers to produce low-zinc breast milk and thus may be involved in TNZD pathogenesis in breast-fed infants. For another 27 SNP mutants, we found minimal defects in the cell viability assay (see Supplementary 
a

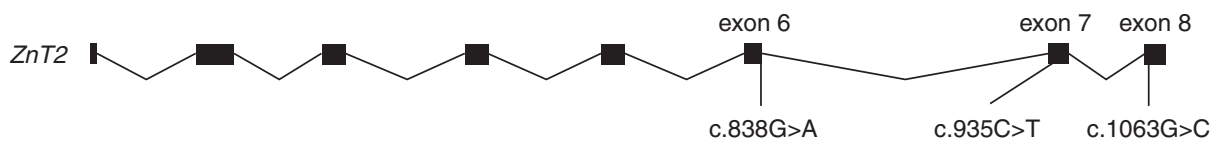

b

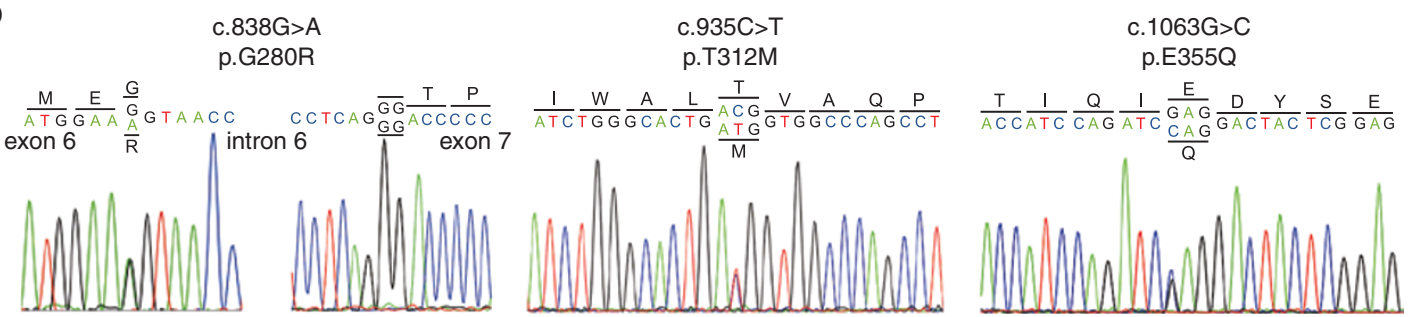

Figure 1. Three novel missense SLC3OA2/ZnT2 mutations in Japanese mothers of transient neonatal zinc-deficient infants. (a) Schematic of SLC30A2/ZnT2 and positions of mutations found in this study. Three novel missense mutations, c.838G $>A$ in exon 6, c.935C $>T$ in exon 7, and c.1063G $>C$ in exon 8, were identified in three mothers. (b) Electropherograms showing the three novel SLC30A2/ZnT2 mutations. The c.838G $>A$ mutation (left panel) was found at the $3^{\prime}$ end of exon 6, and thus, the last G or A nucleotide in exon 6 and the first GG nucleotides in exon 7 generate a GGG or AGG codon, respectively.

a

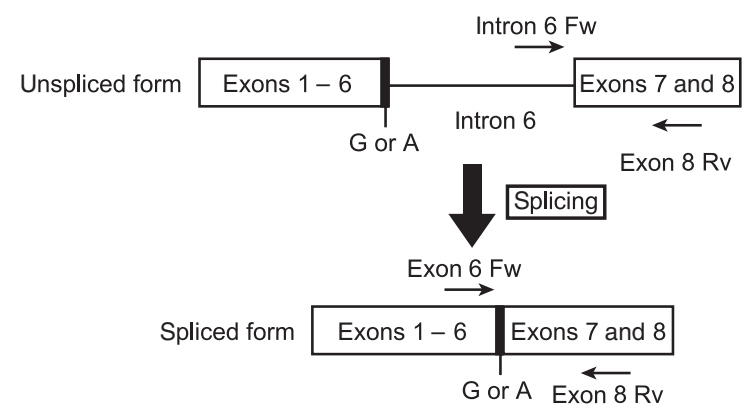

b

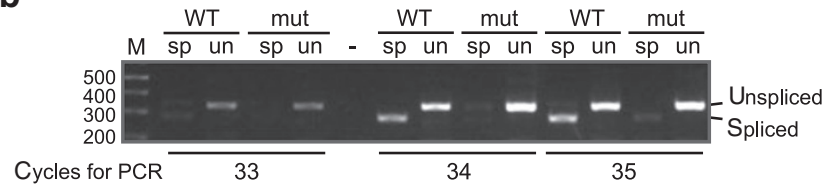

Figure 2. c.838G $>$ A mutation reduced the splicing efficiency of intron 6 in the $Z n T 2$ transcript. (a) Schematic representation of $Z n T 2$ transcripts detected in $b$, in which the nucleotide at the end of exon 6 is $G$ (wild type, WT) or A (mutant). ZnT2 cDNA fragments containing the unspliced or the spliced form of intron 6 were amplified. Arrows indicate the position of primers used. (b) Results of semiquantitative reverse transcriptase-PCR. PCR products amplified after 33-35 cycles were quantified by densitometric analysis, and the relative intensity of the spliced form to the unspliced form was calculated as 0.59 and 0.15 for WT and mutant transcripts, respectively, at cycle 34 . The panel shows representative results of triplicate experiments. -, PCR blank.

Table S3 online); therefore, these SNPs are unlikely to be involved in TNZD pathogenesis.

\section{DISCUSSION}

We are aware of at least 20 different reports and abstracts of transient zinc deficiency in full-term breast-fed infants published in the past 8 y (2007-2014) in Japan (see Supplementary Table S1 online). Moreover, 17 case reports of this condition were presented in domestic Japanese pediatrics and dermatology journals between 1981 and
2006 (28). In all cases, zinc deficiency was attributed to low levels of zinc in the mother's breast milk, and symptoms were cured by zinc supplementation therapy and did not reoccur, which is typical for TNZD. Considering the paucity of TNZD-related publications in international journals (24), the frequency of reported TNZD cases in Japan is of interest.

At present, over $50 \%$ of mothers in Japan are estimated to be exclusively breast-feeding their infants, which is a significant increase compared with the number in 2000 (about 40\%) (29). This may explain the recent increase in case reports, because the use of fortified formulas would mask the progression of TNZD and impact on apparent rates of TNZD. Additionally, pediatricians in Japan have recently learned that infant dermatitis is caused by zinc deficiency (30). In most cases, TNZD is first suspected by low alkaline phosphatase activity $(28,31,32)$, which is routinely measured as a marker of liver function, and which is significantly dependent on zinc levels (33). In infants where low alkaline phosphatase activity is detected, prompt measurement of zinc levels in the infant's serum and the mother's breast milk would be useful to facilitate early TNZD diagnosis.

In this study, we identified three novel, heterozygous SLC30A2/ZnT2 mutations in three affected Japanese mothers: c.838G $>\mathrm{A}$ in exon $6, \mathrm{c} .935 \mathrm{C}>\mathrm{T}$ in exon 7 , and c.1063G $>\mathrm{C}$ in exon 8 . The c.838G $>\mathrm{A}$ mutation reduced splicing efficiency, and also caused a G280R substitution, which resulted in the loss of zinc-transport activity. Moreover, T312M (c.935C > T) and E355Q (c.1063G>C) substitutions in SLC30A2/ZnT2 resulted in loss-of-function. The three novel TNZD-causing mutations presented here bring the total of TNZD-causing mutations identified to date to eight. There is, therefore, a larger variety, and a higher frequency, of TNZD-causing SLC30A2/ ZnT2 mutations than previously thought. Including the three cases in this study, all cases have reported heterozygous mutations in SLC30A2/ZnT2, except for one case with compound heterozygous mutations (13), suggesting that TNZD is caused 
a

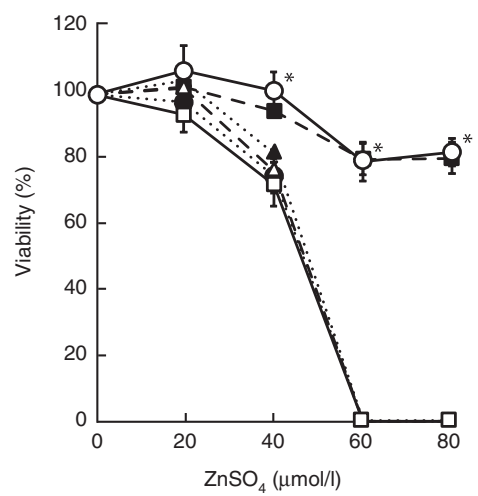

C
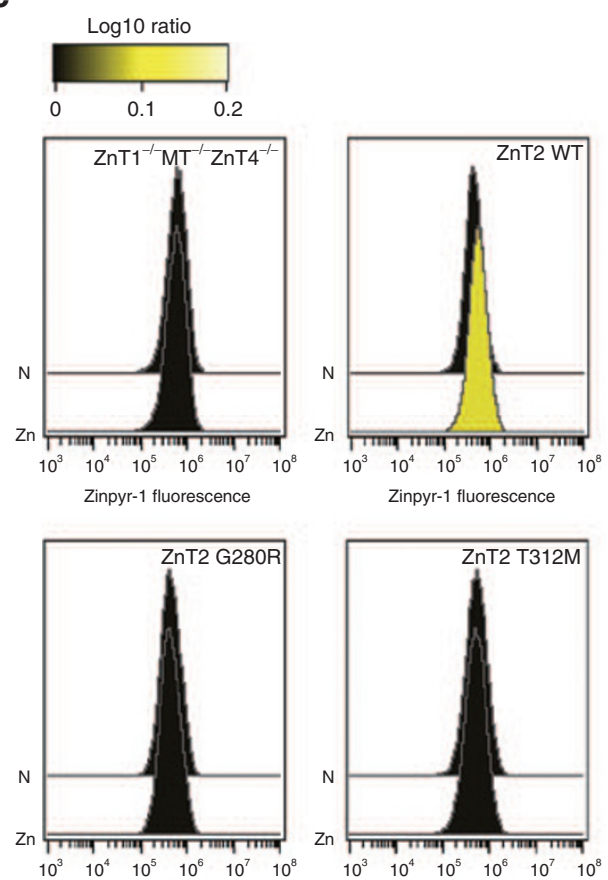

Zinpyr-1 fluorescence

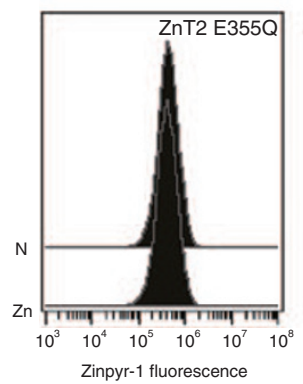

b

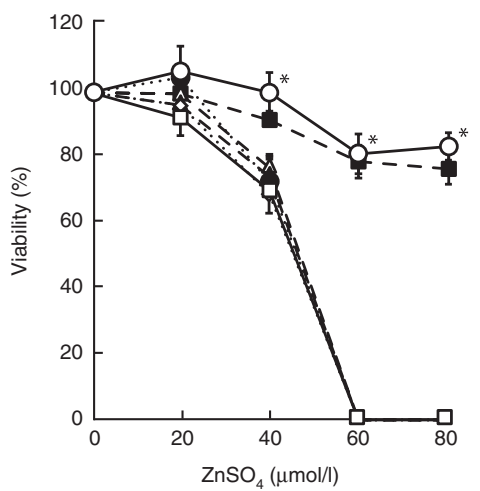

d
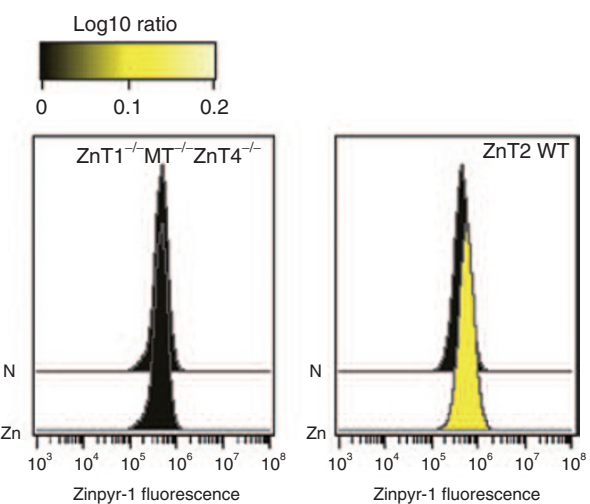

Zinpyr-1 fluorescence

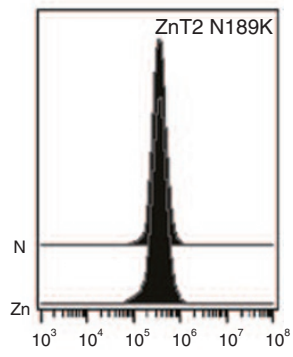

Zinpyr-1 fluorescence
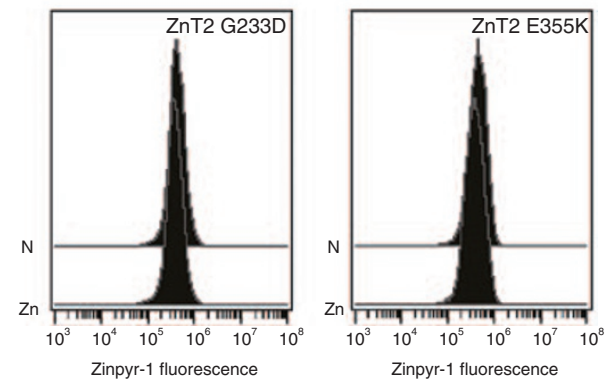

Figure 3. Evaluation of zinc-transport activity of ZnT2 mutants. Zinc-transport activity of the indicated ZnT2 proteins was determined based on a viability test of zinc-sensitive cells incubated in high extracellular zinc concentrations and a fluorescent zinc-selective probe staining. (a) Evaluation of

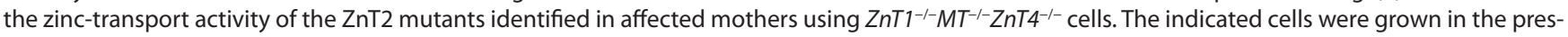
ence of indicated concentrations of $\mathrm{ZnSO}_{4}$ for $72 \mathrm{~h}$, and the number of living cells was measured using the alamarBlue assay (plotted as a percentage of

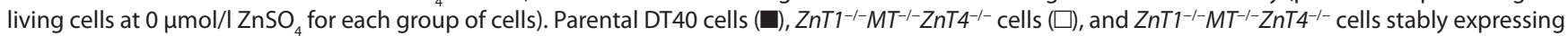

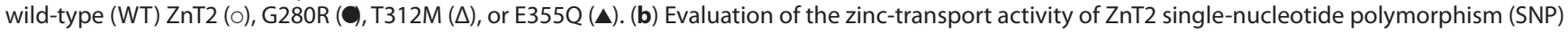
mutants using $\mathrm{ZnT}^{-/-} \mathrm{MT}^{-/-} \mathrm{ZnT}_{4}{ }^{-/}$cells, as described in a. Cells stably expressing each of the ZnT2 SNP mutants were examined, but only results of the four SNP mutants whose zinc-transport activity was measured as null are shown. Parental DT40 cells $(\square), Z n T 1^{---} M T^{-1-} \mathrm{ZnT4^{-/- }}$ cells $(\square)$, and

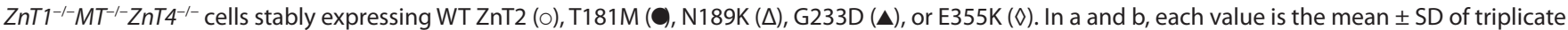
experiments. *A significant difference between the cells expressing WT ZnT2 and mutant ZnT2 proteins $\left({ }^{*} P<0.05\right)$. (c, d) Evaluation of the zinc-transport activity of $\mathrm{ZnT2}$ mutants examined in a and b using a fluorescent zinc-selective probe staining. The cells were grown with or without $30 \mu \mathrm{mol} / \mathrm{I} \mathrm{ZnSO} \mathrm{O}_{4}$ for $48 \mathrm{~h}$ and then loaded with Zinpyr-1. After washing, the cells were analyzed by flow cytometry. Each histogram represents 50,000 cells and indicates zinc levels detected by Zinpyr-1 fluorescence. 


\section{Articles $\mid$ Istumurae tal.}

a
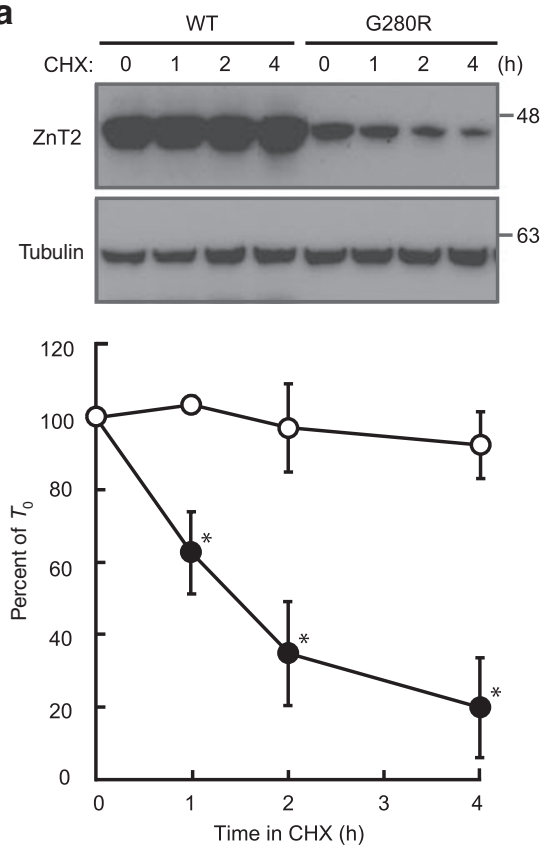

b

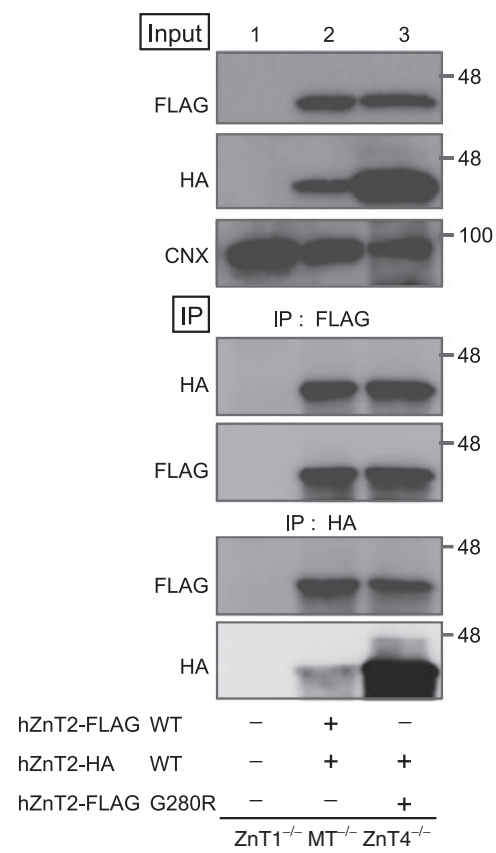

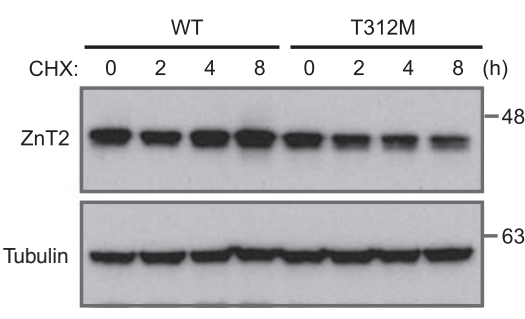
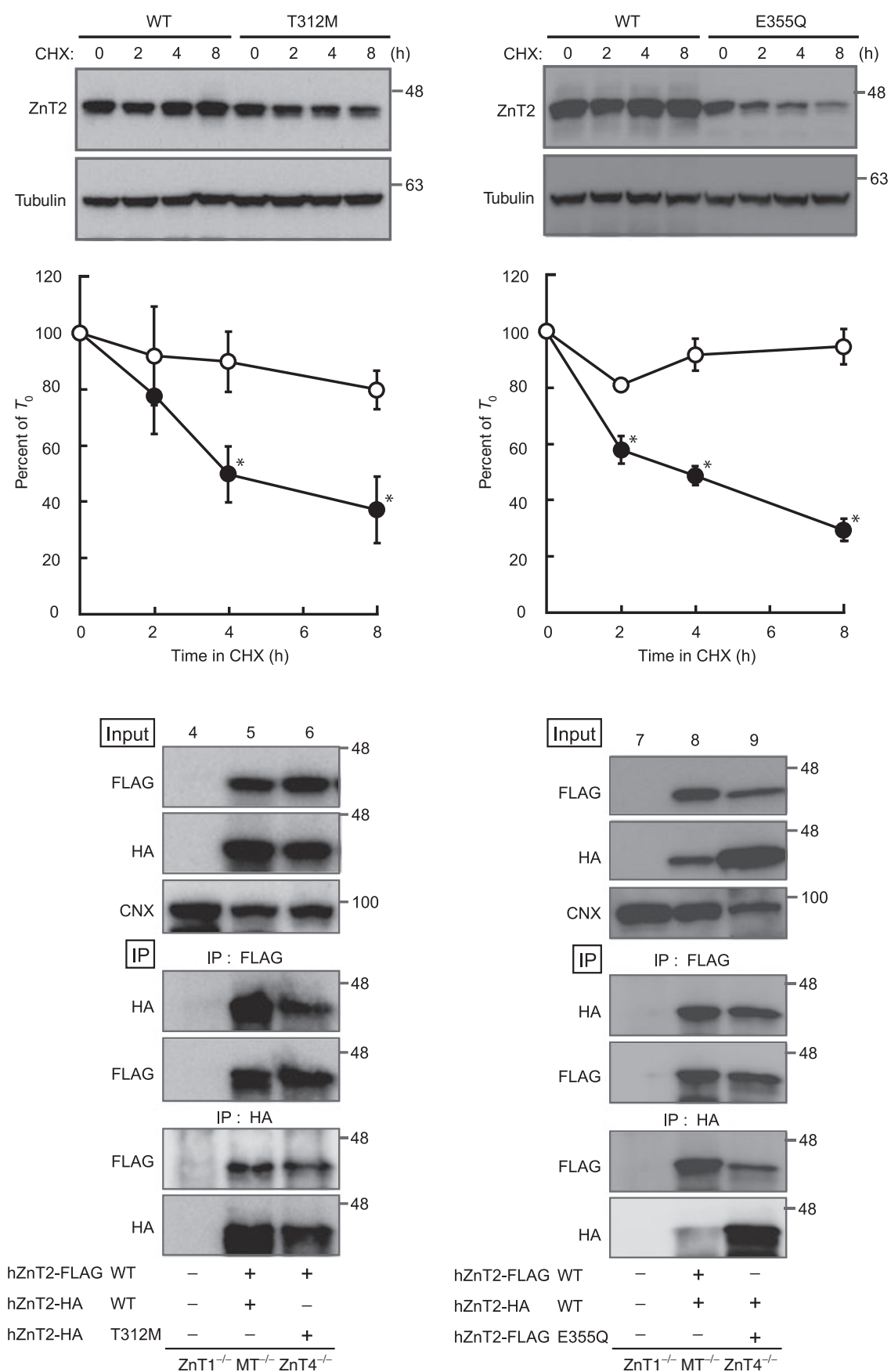

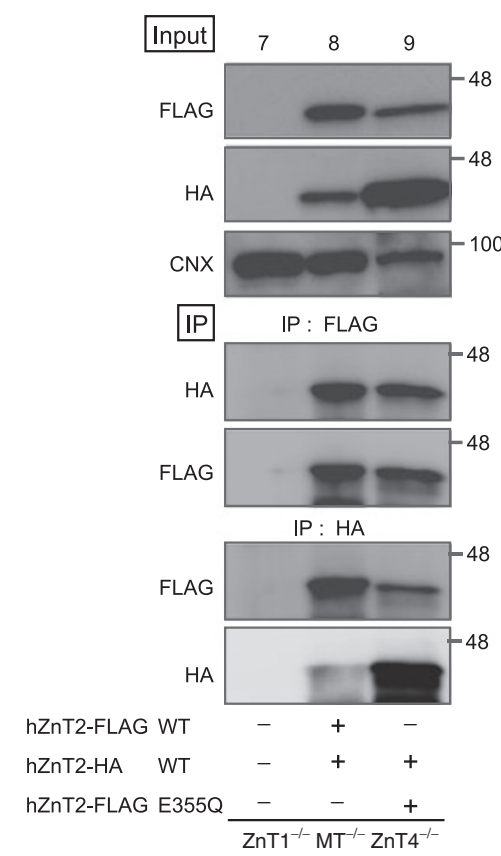

Figure 4. Evaluation of protein stability and dimerization of ZnT2 mutants found in three Japanese mothers of transient neonatal zinc-deficient infants. (a) Evaluation of protein stability of G280R, T312M, and E355Q ZnT2 mutants. The expression levels of the wild-type (WT) (o) and each mutant (C) ZnT2 protein at each time point are shown. The collection of cells at $8 \mathrm{~h}$ was performed when the band intensity of the ZnT2 mutant protein was more than $50 \%$ at $2 \mathrm{~h}$ after cycloheximide treatment, compared with that at $0 \mathrm{~h}$. Representative results of immunoblot analysis to monitor ZnT2 levels are shown (upper panel). Each value is the mean \pm SD of triplicate experiments (lower panel). ${ }^{*} \mathrm{~A}$ significant difference between levels of the WT and mutant ZnT2 proteins at each time point $\left({ }^{*} P<0.05\right)$. Tubulin is shown as a loading control. (b) Evaluation of dimerization of G280R, T312M, and E355Q ZnT2 mutants. Tagged-ZnT2 WT or mutants were immunoprecipitated with antibodies against either the FLAG or HA epitopes. The immunoprecipitates were analyzed by immunoblotting using antibodies against the FLAG or HA tags. To estimate the amount of tagged ZnT2 WT and mutant proteins, $10 \%$ of each aliquot was subjected to immunoblot analysis (input panels). Calnexin (CNX) is shown as a loading control. The panels show representative results.

by haploinsufficiency or dominant negative mechanisms $(10,12-15)$.

Consistent with affected mothers in previous reports $(10,12-15)$, the three affected mothers in this study produced breast milk with $>70 \%$ reduced zinc levels (see
Supplementary Table S2 online). Moreover, in the 20 recent reports and abstracts of TNZD in Japan, breast milk zinc levels were decreased by $>60 \%$ in all mothers (see Supplementary Table S1 online). However, comparison of zinc levels at the time of TNZD diagnosis with average normal zinc levels at 
Novel TNZD-causing mutations in SLC30A2

the corresponding time point during lactation is unreliable for setting a threshold for TNZD pathogenesis, because zinc levels in breast milk vary considerably among mothers $(34,35)$. Furthermore, the onset or progression of disease is likely to depend on the health condition of the infant, including the zinc pool in the body. Thus, the relationship between zinc levels in breast milk and the onset and progression of TNZD needs to be more extensively investigated in future studies. In the 20 recent reports and abstracts, the maximum zinc level was $32 \mu \mathrm{g} / \mathrm{dl}$ at 5 mo (see Supplementary Table S1 online). Accordingly, a breast milk zinc content of $32 \mu \mathrm{g} / \mathrm{dl}$ or lower may be an index for the onset and progression of TNZD symptoms.

Many SNPs have been identified in SLC30A2/ZnT2, and several have been well characterized; for example, two SNPs, rs35235055 (c.68T >C causing L23P) and rs35623192 (c.1018C > T causing R340C), have been proposed to compromise zinc secretion in breast milk (36). In this study, we identified a further four SNPs that result in the loss of zinc-transport activity, suggesting their potential involvement in low levels of zinc secretion into milk. Of these, the position of rs377192955 (c.1063G >A causing E355K) is identical to that of the TNZD mutation found in patient 3 (E355Q), indicating that our SNP evaluation yielded reliable results. Because the molecular basis of ZnT2 functions has recently been revealed $(37,38)$, detailed characterization of each SLC30A2/ZnT2 SNP and the relationship between each SLC30A2/ZnT2 SNP and the effects on zinc-transport activity would be beneficial for understanding TNZD pathogenesis. Further research is needed to determine if SLC30A2/ZnT2 SNPs can cause subtler or minor adverse effects.

In conclusion, infant zinc nutrition is of particular importance for healthy growth and development, and zinc levels in breast milk should be of primary concern. TNZD occurs through a deficiency of zinc levels in the mother's breast milk, which is controlled by ZnT2. Determination of the TNZD frequency is difficult to estimate because it may be masked by the use of fortified formulas. Moreover, there is a paucity of genetic information concerning TNZD pathogenesis (24). However, our present results provide crucial genetic information about TNZD pathogenesis and indicate that the frequency of TNZD may be higher than previously thought. These findings provide helpful information to support the normal growth and development of breast-fed infants.

\section{METHODS}

Three full-term breast-fed infant patients were diagnosed with zinc deficiency by their pediatricians based on clinical presentations. Each infant showed low serum zinc levels and presented with symptoms of zinc deficiency at 1.5-5 mo. Zinc levels in the breast milk of all three mothers were also lower than the normal level expected during lactation $(35,39)$; however, their serum zinc levels were normal. Patient 1 was a full-term female (gestational age, $40 \mathrm{wk}$; birth weight, 2,630 g), who had been exclusively fed on breast milk from her mother. Dermatitis was present from 3 mo of age. Her serum zinc level was significantly low $(13 \mu \mathrm{g} / \mathrm{dl}$ at $4 \mathrm{mo}$; normal level: $70-120 \mu \mathrm{g} /$ dl). At $4 \mathrm{mo}$, her mother's breast milk zinc level of $10 \mu \mathrm{g} / \mathrm{dl}$ was lower than the normal level of $80 \pm 30 \mu \mathrm{g} / \mathrm{dl}$ at 4-6 mo. Clinical information for patients 2 and 3 has been reported in domestic Japanese journals $(31,32)$, and the clinical data of all patients are summarized in
Table 1. SNPs in SLC3OA2/ZnT2 that cause loss of zinc-transport activity

\begin{tabular}{lcccc}
\hline $\begin{array}{l}\text { Reference SNP } \\
\text { ID number }\end{array}$ & Substitution & Protein & Exon & $\begin{array}{c}\text { Global minor } \\
\text { allele frequency }\end{array}$ \\
\hline rs148861822 & c.542C>T & T181M & 4 & N.A. \\
rs200520278 & c.567C >A & N189K & 4 & A $=0.0002$ \\
rs201084300 & c.698G >A & G233D & 5 & A $=0.0002$ \\
rs377192955 & c.1063G >A & E355K & 8 & N.A. \\
\hline
\end{tabular}

N.A., not applicable; SNP, single-nucleotide polymorphism.

a1000 Genome phase III, genotype data from 2,504 worldwide individuals.

Supplementary Table S2 online. All infants were given Polaprezinc (INN: $\left(\mathrm{C}_{9} \mathrm{H}_{12} \mathrm{~N}_{4} \mathrm{O}_{3} \mathrm{Zn}\right)_{n}$, Zeria Pharmaceutical,Tokyo, Japan) oral zinc replacement therapy, which promptly eliminated symptoms.

\section{Sequencing SLC3OA2/ZnT2}

Genomic DNA was isolated from the mothers' whole blood using a QIAamp DNA Blood Midi Kit (Qiagen, Hilden, Germany) or a NucleoSpin Blood Kit (Macherey-Nagel, Düren, Germany). All exons containing coding regions (including splice sites) of $S L C 30 A 2 / Z n T 2$, and the sequence in and around the promoter regions of SLC3OA2/ $\mathrm{ZnT} 2$ were directly sequenced in both directions, using fragments amplified from the isolated genomic DNA. Primer information is described in our previous study (13).

Plasmid Construction, and Transient and Stable Transfection ZnT2 cDNA expression plasmids containing intron 6, with G (WT) or A (mutant) at position c. 838 at the $3^{\prime}$ end of exon 6 were constructed (see Figure 2a). DT40 cells were transiently transfected with each plasmid as described previously (40). For normalization of transient transfection efficiency, Renilla luciferase was used, and the activity ratio of each transfection was within 1.15. To evaluate zinc-transport activity in each ZnT2 mutant in stable transformants, we introduced mutations into $Z n T 2$ cDNA as described previously (13). DNA transfection to establish cells stably expressing WT ZnT2 or mutant ZnT2 was performed by electroporation as described previously $(13,40)$.

\section{Reverse Transcriptase-PCR Analysis}

Total RNA was isolated from transiently transfected DT40 cells using Sepasol-RNA I Super (Nacalai Tesque, Kyoto, Japan). Reverse transcription was performed using ReverTra Ace (Toyobo, Osaka, Japan) and 1 $\mu \mathrm{g}$ total RNA as a template. PCR was performed using KOD Plus polymerase (Toyobo). The amplified products of spliced ( $281 \mathrm{bp}$ ) or unspliced (330 bp) forms of ZnT2 mRNA were electrophoresed on a 3\% agarose gel and stained with ethidium bromide. For semiquantitative reverse transcriptase-PCR, products amplified after 33-35 cycles were quantified by densitometry using ImageQuant (GE Healthcare, Waukesha, WI). Primer sequences are listed in Supplementary Table S4 online.

Evaluation of Zinc-Transport Activity of ZnT2 Proteins Based on a Viability Test of Zinc-Sensitive Cells Against High Extracellular Zinc Concentrations

DT40 cells deficient in ZnT1, ZnT4, and metallothionein genes (MT) $\left(Z n T 1^{-1-} M T^{-1-} Z n T 4^{-/-}\right.$cells) were cultured as described previously $(13,40) . Z n T 1^{-1-} M T^{-1-} Z n T 4^{-/-}$cells fail to grow in the presence of $60 \mu \mathrm{mol} / \mathrm{l}$ or more $\mathrm{ZnSO}_{4}$, whereas $Z n T 1^{-1-} M T^{-1-} Z n T 4^{---}$cells stably expressing zinc-transport competent ZnT2 can grow in a similar manner to parental cells. Thus, cell viability in high levels of extracellular zinc reflects the zinc-transport activity of ZnT2 $(13,40)$. Cell viability was determined using the alamarBlue assay (Trek Diagnostic Systems, Westlake, $\mathrm{OH}$ ).

\section{Evaluation of Zinc-Transport Activity of ZnT2 Proteins Using a Fluorescent Zinc-Selective Probe and Flow Cytometry}

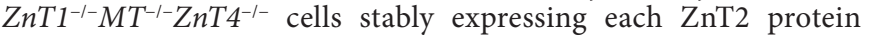
were grown in the presence or absence of $30 \mu \mathrm{mol} / \mathrm{ZnSO}_{4}$ for $48 \mathrm{~h}$ and washed once in phosphate-buffered saline. Cells were then treated with $5 \mu \mathrm{mol} / \mathrm{l}$ Zinpyr-1 ester (Santa Cruz Biotechnology, Santa Cruz, CA) for $30 \mathrm{~min}$ at room temperature. After extensive 
a
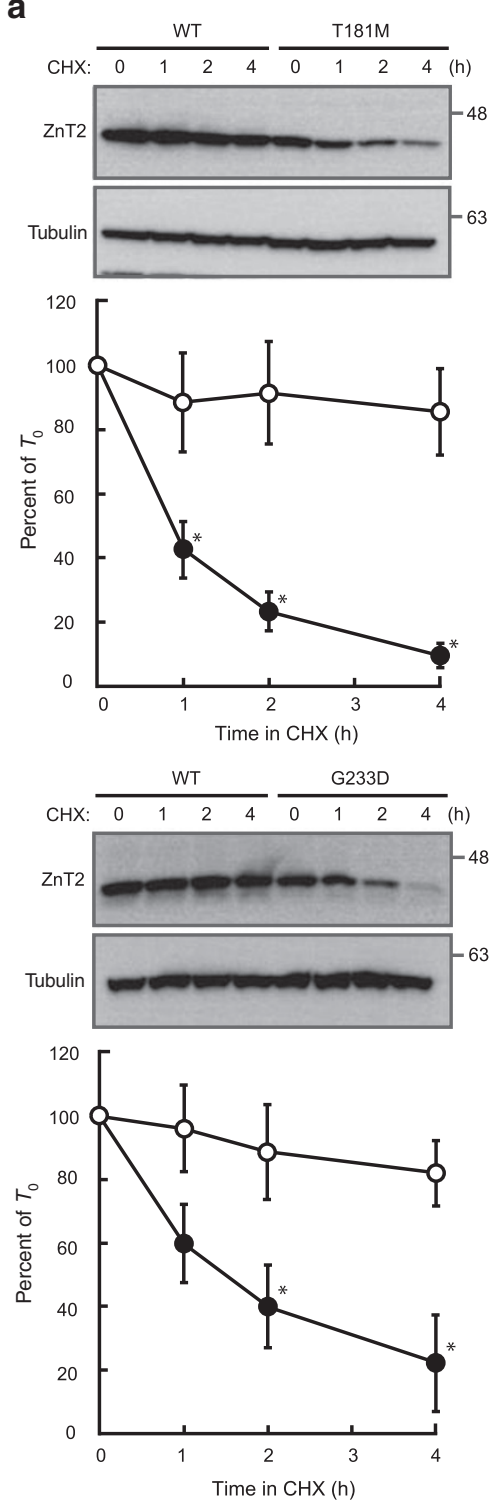
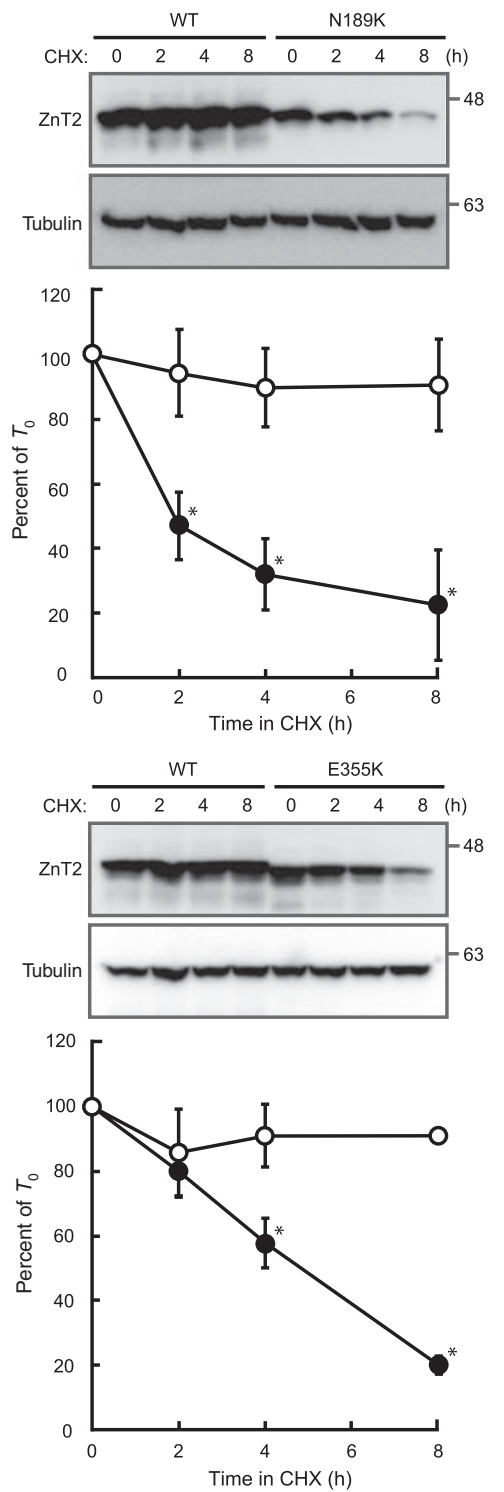

b
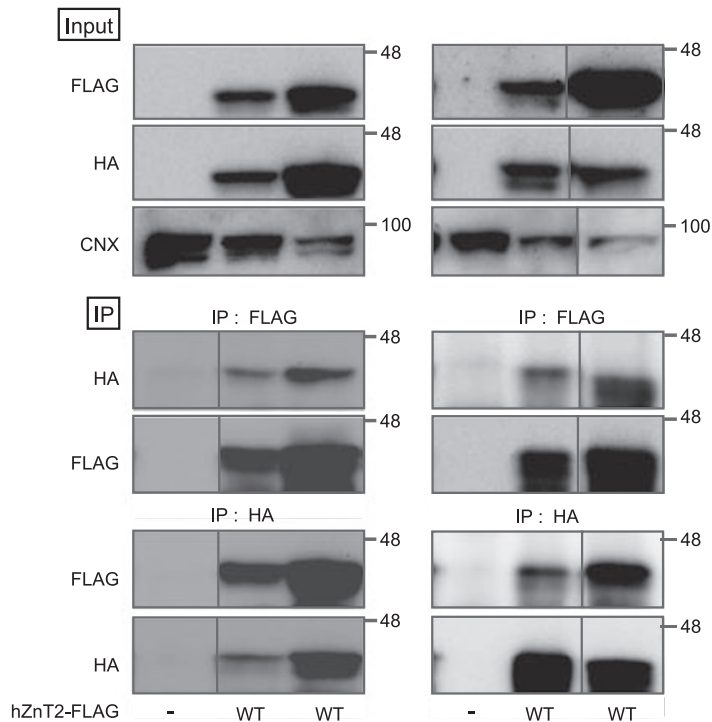

IP : HA

hZnT2-HA

$\begin{array}{ccc}- & \text { WT } & \text { WT } \\ - & \text { T181M } & \text { N189K }\end{array}$

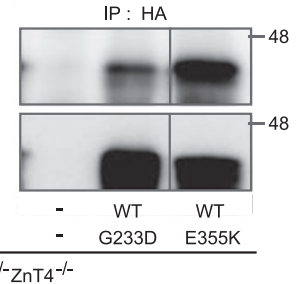

$\mathrm{ZnT}^{-/-} \mathrm{MT}^{-/-} \mathrm{ZnT} 4^{-1-}$

Figure 5. Evaluation of protein stability and dimerization of ZnT2 single-nucleotide polymorphism (SNP) mutants that lack zinc-transport activity. (a) Evaluation of the protein stability of T181M (rs148861822), N189K (rs200520278), G233D (rs201084300), and E355K (rs377192955) was performed as in Figure 4. The expression levels of the wild-type (WT) (0) and each mutant ( $\mathrm{ZnT2}$ protein at each time point are shown. Representative results of immunoblot analysis to monitor ZnT2 levels are shown (upper panel). Each value is the mean \pm SD of triplicate experiments (lower panel). ${ }^{*} \mathrm{~A}$ significant difference between levels of the WT and mutant ZnT2 proteins at each time point $\left({ }^{*} P<0.05\right)$. Tubulin is shown as a loading control. (b) Evaluation of the dimerization of T181M, N189K, G233D, and E355K ZnT2 mutants. Tagged-ZnT2 WT or mutants were immunoprecipitated with antibodies against either the FLAG or HA epitopes. The immunoprecipitates were analyzed by immunoblotting using antibodies against the FLAG or HA tags. To estimate the amount of tagged ZnT2 WT and mutant proteins, $10 \%$ of each aliquot was subjected to immunoblot analysis (input panels). The noncontiguous boxed lanes were derived from the same blot. Calnexin (CNX) is shown as a loading control. The panels show representative results.

washing with phosphate-buffered saline containing $20 \mathrm{mmol} / \mathrm{l}$ ethylenediaminetetraacetic acid to remove extracellular zinc, the cells were resuspended in phosphate-buffered saline containing $1 \%$ bovine serum albumin and were subjected to flow cytometric analysis using a BD Accuri C6 flow cytometer (BD Biosciences, Ann Arbor, MI). Histograms were overlaid according to the $\log 10$ ratio increase of Zinpyr-1 fluorescence in zinc-supplemented (30 $\mu \mathrm{mol} / \mathrm{l} \mathrm{ZnSO}_{4}$ ) conditions relative to normal conditions using Cytobank (http://www.cytobank.org/). The fluorescence intensity of Zinpyr-1 increases with zinc accumulation in vesicles and intracellular compartments mobilized from the cytosol and thus reflects the zinc-transport activity of ZnT2 proteins expressed in

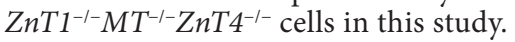

\section{Evaluation of Stability of Mutant ZnT2 Proteins}

Cells expressing WT or mutant ZnT2 were treated with cycloheximide to block further protein synthesis and collected periodically over $4-8 \mathrm{~h}$. The collection of cells at $8 \mathrm{~h}$ was performed when the band intensity of the ZnT2 mutant protein was more than $50 \%$ at $2 \mathrm{~h}$ after treatment, compared with that at $0 \mathrm{~h}$. Total cell lysates were prepared from cells and subjected to immunoblotting to monitor ZnT2 levels as described previously (13). The following antibodies were used: anti-ZnT2 (1:4,000 dilution) (13) or anti-tubulin (1:20,000; Sigma, St. Louis, MO). The ZnT2 band intensities are the averages of three independent experiments and are shown as the percentage of the intensity at $0 \mathrm{~h}\left(T_{0}\right)$ after normalization against tubulin at each time point. Fluoroimages were obtained using a LAS1000 Plus image analyzer 


\section{Novel TNZD-causing mutations in SLC30A2}

(Fujifilm, Tokyo, Japan). Densitometry quantification was performed using ImageQuant (GE Healthcare).

\section{Evaluation of Dimerization by Immunoprecipitation}

Dimerization of ZnT2 was examined by immunoprecipitation as described previously (13). Briefly, membrane fractions prepared from cells stably expressing both HA- or FLAG-tagged WT and mutant ZnT2 were incubated with anti-FLAG M2 (1:200 dilution; Sigma) or anti-HA HA-11 (1:200 dilution; Covance, Emeryville, CA) antibodies in NP-40 buffer for $1 \mathrm{~h}$. Then, $10 \mu \mathrm{l}$ of Protein G-Sepharose beads (GE Healthcare) were added and incubated for $2 \mathrm{~h}$. Immunoprecipitates were then subjected to immunoblotting with polyclonal anti-HA (1:4,000; MBL, Nagoya, Japan) or polyclonal anti-FLAG (antiDDDDK; 1:4,000; MBL) antibodies. To estimate the amount of WT and mutant ZnT2 proteins in samples, $10 \%$ of each aliquot was subjected to immunoblot analysis (input panels) using anti-FLAG M2 (1:4,000 dilution), anti-HA HA-11 (1:4,000 dilution) or anti-calnexin (1:4,000 dilution; Stressgen, Ann Arbor, MI) antibodies. Experiments were performed three times, giving similar results.

\section{Statistical Analyses}

All data are depicted as the mean \pm SD. Statistical significance was determined by the Student's $t$-test and accepted at $P<0.05$.

\section{Ethics Approval}

This study was approved by the Institutional Review Board of Teikyo University School of Medicine (No. 09-066) and by the Ethics Committee of Kyoto University Graduate School and Faculty of Medicine (Nos. G532 and G573). Written consent was obtained from each patient's mother.

\section{SUPPLEMENTARY MATERIAL}

Supplementary material is linked to the online version of the paper at http:// www.nature.com/pr

\section{ACKNOWLEDGMENTS}

We thank the patients and their families for their interest and cooperation in this study.

\section{STATEMENT OF FINANCIAL SUPPORT}

This work was supported by Grants-in-Aid for Challenging Exploratory Research and Scientific Research (B) from the Japan Society for the Promotion of Science (KAKENHI, Grant numbers 26660086 and 15H04501), the Fuji Foundation for Protein Research (Osaka, Japan), the Foundation for Dietary Scientific Research (Tokyo, Japan), and the Morinaga Foundation for Health and Nutrition (Tokyo, Japan) (to T.K.). N.I. is a Research Fellow (DC2) of the Japan Society for the Promotion of Science.

Disclosure: There is no conflict of interest to disclose.

\section{REFERENCES}

1. Kambe T, Tsuji T, Hashimoto A, Itsumura N. The physiological, biochemical, and molecular roles of zinc transporters in zinc homeostasis and metabolism. Physiol Rev 2015;95:749-84.

2. Fukada T, Kambe T. Molecular and genetic features of zinc transporters in physiology and pathogenesis. Metallomics 2011;3:662-74.

3. Hambidge KM, Krebs NF, Westcott JE, Miller LV. Changes in zinc absorption during development. J Pediatr 2006;149:5 Suppl:S64-8.

4. Krebs NF. Update on zinc deficiency and excess in clinical pediatric practice. Ann Nutr Metab 2013;62:Suppl 1:19-29.

5. Kelleher SL, Seo YA, Lopez V. Mammary gland zinc metabolism: regulation and dysregulation. Genes Nutr 2009;4:83-94.

6. Kelleher SL, McCormick NH, Velasquez V, Lopez V. Zinc in specialized secretory tissues: roles in the pancreas, prostate, and mammary gland. Adv Nutr 2011;2:101-11.

7. Donangelo CM, King JC. Maternal zinc intakes and homeostatic adjustments during pregnancy and lactation. Nutrients 2012;4:782-98.

8. Kelleher SL, Lönnerdal B. Molecular regulation of milk trace mineral homeostasis. Mol Aspects Med 2005;26:328-39.

9. Roberts LJ, Shadwick CF, Bergstresser PR. Zinc deficiency in two full-term breast-fed infants. J Am Acad Dermatol 1987;16:2 Pt 1:301-4.
10. Chowanadisai W, Lönnerdal B, Kelleher SL. Identification of a mutation in SLC30A2 (ZnT-2) in women with low milk zinc concentration that results in transient neonatal zinc deficiency. J Biol Chem 2006;281:39699-707.

11. Murthy SC, Udagani MM, Badakali AV, Yelameli BC. Symptomatic zinc deficiency in a full-term breast-fed infant. Dermatol Online J 2010;16:3.

12. Lasry I, Seo YA, Ityel H, et al. A dominant negative heterozygous G87R mutation in the zinc transporter, ZnT-2 (SLC30A2), results in transient neonatal zinc deficiency. J Biol Chem 2012;287:29348-61.

13. Itsumura $\mathrm{N}$, Inamo $\mathrm{Y}$, Okazaki $\mathrm{F}$, et al. Compound heterozygous mutations in SLC30A2/ZnT2 results in low milk zinc concentrations: a novel mechanism for zinc deficiency in a breast-fed infant. PLoS One 2013;8:e64045.

14. Miletta MC, Bieri A, Kernland K, et al. Transient neonatal zinc deficiency caused by a heterozygous G87R mutation in the zinc transporter ZnT-2 (SLC30A2) gene in the mother highlighting the importance of $\mathrm{Zn}(2+)$ for normal growth and development. Int J Endocrinol 2013;2013:259189.

15. Lova Navarro M, Vera Casaño A, Benito López C, et al. Transient neonatal zinc deficiency due to a new autosomal dominant mutation in gene SLC30A2 (ZnT-2). Pediatr Dermatol 2014;31:251-2.

16. Kambe T, Hashimoto A, Fujimoto S. Current understanding of ZIP and ZnT zinc transporters in human health and diseases. Cell Mol Life Sci 2014;71:3281-95.

17. Kasana S, Din J, Maret W. Genetic causes and gene-nutrient interactions in mammalian zinc deficiencies: acrodermatitis enteropathica and transient neonatal zinc deficiency as examples. J Trace Elem Med Biol 2015; 29:47-62.

18. Kienast A, Roth B, Bossier C, Hojabri C, Hoeger PH. Zinc-deficiency dermatitis in breast-fed infants. Eur J Pediatr 2007;166:189-94.

19. Ackland ML, Michalczyk A. Zinc deficiency and its inherited disorders-a review. Genes Nutr 2006;1:41-9.

20. Aggett PJ, Atherton DJ, More J, Davey J, Delves HT, Harries JT. Symptomatic zinc deficiency in a breast-fed preterm infant. Arch Dis Child 1980;55:547-50.

21. Barbarot S, Chantier E, Kuster A, et al. Symptomatic acquired zinc deficiency in at-risk premature infants: high dose preventive supplementation is necessary. Pediatr Dermatol 2010;27:380-3.

22. Kambe T, Weaver BP, Andrews GK. The genetics of essential metal homeostasis during development. Genesis 2008;46:214-28.

23. Cheng HC, Wang JD, Chen CH, Yang CS. A young infant with periorificial and acral dermatitis. J Pediatr 2014;165:408-408.e1.

24. Weaver BP, Andrews GK. Zinc transporter mutations and human growth. In: Preedy VR, ed. Handbook of Growth and Growth Monitoring in Health and Disease. Berlin, Germany: Springer Science + Business Media, LLC, 2012:2319-36.

25. Turunen JJ, Niemelä EH, Verma B, Frilander MJ. The significant other: splicing by the minor spliceosome. Wiley Interdiscip Rev RNA 2013;4:6176.

26. Palmiter RD, Cole TB, Findley SD. ZnT-2, a mammalian protein that confers resistance to zinc by facilitating vesicular sequestration. EMBO J 1996;15:1784-91.

27. Woodroofe CC, Masalha R, Barnes KR, Frederickson CJ, Lippard SJ. Membrane-permeable and -impermeable sensors of the Zinpyr family and their application to imaging of hippocampal zinc in vivo. Chem Biol 2004;11:1659-66.

28. Hirabe C, Hosokawa C, Takahara M, et al. A case of acquired zinc deficiency due to low zinc concentration in maternal breast milk. Nishinihon J Dermatol 2008;70:402-5.

29. Ministry of Health, Labour and Welfare. National nutrition survey on preschool children in Japan, 2010. (http://www.mhlw.go.jp/toukei/list/83-1. html.)

30. Kodama H, Ogawa E, Motoyama K, Sato Y. Zinc deficiency in infants and adults. Pediatrics of Japan 2014;55:341-8.

31. Okumura S, Terakawa T, Yokomichi S, Miyata A. My experience: case of acquired zinc deficiency in full term breast-fed infant. J Ambul Gen Pediatr 2009;12:221-5.

32. Yasuike R, Okuzawa Y, Tamagawa-Mineoka R, Nakajima H, Katoh N. A case of zinc deficiency due to low zinc concentration in maternal breast milk. J Pediat Dermatol 2013;32:153-6. 


\section{Articles | Itsumura et al.}

33. Kambe T. An overview of a wide range of functions of $\mathrm{ZnT}$ and Zip zinc transporters in the secretory pathway. Biosci Biotechnol Biochem 2011;75: 1036-43.

34. Yamawaki N, Yamada M, Kan-no T, Kojima T, Kaneko T, Yonekubo A. Macronutrient, mineral and trace element composition of breast milk from Japanese women. J Trace Elem Med Biol 2005;19:171-81.

35. Dórea JG. Zinc deficiency in nursing infants. J Am Coll Nutr 2002;21: 84-7.

36. Seo YA, Kelleher SL. Functional analysis of two single nucleotide polymorphisms in SLC30A2 (ZnT2): implications for mammary gland function and breast disease in women. Physiol Genomics 2010;42A: 219-27.
37. Hennigar SR, Seo YA, Sharma S, Soybel DI, Kelleher SL. ZnT2 is a critical mediator of lysosomal-mediated cell death during early mammary gland involution. Sci Rep 2015;5:8033.

38. Lee S, Hennigar SR, Alam S, Nishida K, Kelleher SL. Essential role for zinc transporter 2 ( $\mathrm{ZnT} 2$ )-mediated zinc transport in mammary gland development and function during lactation. J Biol Chem 2015;290:13064-78.

39. Krebs NF, Reidinger CJ, Hartley S, Robertson AD, Hambidge KM. Zinc supplementation during lactation: effects on maternal status and milk zinc concentrations. Am J Clin Nutr 1995;61:1030-6.

40. Fujimoto S, Itsumura N, Tsuji T, et al. Cooperative functions of ZnT1, metallothionein and $\mathrm{ZnT} 4$ in the cytoplasm are required for full activation of TNAP in the early secretory pathway. PLoS One 2013;8:e77445. 